\title{
Large Orders in the $1 / N$ Perturbation Theory by Inverse Scattering in One Dimension
}

\author{
H. J. de Vega
}

CEN-Saclay, F-91190 Gif-sur-Yvette, France

\begin{abstract}
When one tries to compute large orders in the $1 / N$ series "à la Lipatov" a complicated non-linear equation for the instanton is found in $\phi^{4}$ or non-linear sigma models.

We solve here this equation in the one-dimensional case (quantum mechanics) by inverse scattering techniques. From the instanton solutions we obtain the $K^{\text {th }}$ order of the $1 / N$ perturbation theory up to $0\left(K^{-1}\right)$ for the $0(N)$ symmetric anharmonic oscillator and up to a factor $0\left(K^{0}\right)$ for a non-symmetric model. In the symmetric case we agree with results recently obtained in quantum mechanics by Hikami and Brézin following a different procedure. For the non-symmetric anharmonic oscillator we believe our formulae are new.
\end{abstract}

\section{Introduction}

In the last few years a great attention is paid to perturbative expansions in $1 / N$ in quantum field theory, statistical mechanics and particle physics, $N$ being the size of an internal symmetry group. However little is known in general about the nature of this expansion.

Typically, the $N^{-1}$ series follow by expanding the functional integral in an appropriate representation around some constant stationary point (here labelled "0"). For example in the $N$-component $\phi_{v}^{4}$ theory with lagrangian

$$
L=\frac{1}{2}\left(\partial_{\mu} \vec{\phi}\right)^{2}+\frac{\mu^{2}}{2} \vec{\phi}^{2}+\frac{g \mu^{4-v}}{N}\left(\vec{\phi}^{2}\right)^{2}
$$

the generating functional can be written as (see ref [1] and section II)

$$
\begin{aligned}
& Z(N)=\iint D \alpha(.) \exp (-S[\alpha(\cdot), N] \\
& S=\frac{N}{2} \operatorname{tr} \log \left[-\nabla^{2}+\mu^{2}+4 i \mu^{2-v / 2} \sqrt{\frac{g}{N}} \alpha(\cdot)\right]+\int d^{v} x \alpha(x)^{2} .
\end{aligned}
$$


One gets in this way

$$
Z(N)=\frac{e^{-S_{0}}}{\sqrt{\operatorname{det} M_{0}}} \sum_{K=0}^{\infty} \frac{I_{K}(g)}{N^{K}} .
$$

One can look for the large order behavior of the coefficients $I_{K}$ "à la Lipatov" $[2,3]$. However this leads, even in one-dimension, to a complicated non-linear equation for the instanton that would determine the large $K$ behavior. In the $N$-component $\phi_{v}^{4}$ model considered above, this equation is like

$$
\left\langle\vec{x}\left|\frac{1}{-\nabla^{2}+\mu^{2}+V(\cdot)}\right| \vec{x}\right\rangle=a V(\vec{x})+b
$$

where $\vec{x}=\left(x_{1}, \ldots, x_{v}\right), V(\infty)=0$ and $a, b, \mu^{2}$ are constants. In the $N$-component non-linear sigma model one finds an analogous equation but with $a \equiv 0$. The large orders in $1 / N$ have been calculated by Hikami and Brézin in zero and one dimension for this anharmonic oscillator [4]. In the one-dimensional case they proceed from the Schroedinger equation in spherical coordinates. Because of the $O(N)$ symmetry the problems reduce to large orders in a standard coupling constant in a radial Schroedinger equation where the methods of ref (3) apply.

In the present paper we find explicitly the instantons that rule the large orders in $N^{-1}$ for the $0(N)$-symmetric anharmonic oscillator and also when the quadratic part of the lagrangian is not $O(N)$ symmetric. We do that by inverse scattering methods. This means in our case a change of variables in the functional integral (1.1). There, the functional variable $\alpha(x)$ can be considered the potential $v(x)$ of a one dimensional auxiliar Schroedinger equation times some $x$-independent factor. Then we consider, instead of $v(x)$, the scattering data (SV) associated to it through an auxiliar Schroedinger equation. They are the reflection coefficient, the bound states energies and the normalization constants of the corresponding wave functions [5].

The effective action (1.2) separates completely when expressed in SV. This is not accident and is related to the complete classical integrability of the quadratic oscillator [6]. To get stationary points we look for extrema of the effective action varying the SV. This gives simple linear (algebraic) equations which are immediatly solved (Sect. 2). The instanton so found in the $0(N)$ symmetric model is a reflectionless potential with only one bound state. After computing the small fluctuations around this instanton (Sect. 3) our final result for the $O(N)$ symmetric model reads

where

$$
\begin{aligned}
I_{K}(g)= & \mu \frac{2^{7 / 4}}{\pi^{3 / 2}} \frac{\Gamma\left(K+\frac{1}{2}\right)}{A(g)^{K+1 / 2}} \frac{[3 h(g)-1]^{7 / 4}[\sqrt{3 h(g)-1}-\sqrt{2 h(g)}]}{\sqrt{g[h(g)-1]}[\sqrt{3 h(g)-1}+\sqrt{2 h(g)}]}(-1)^{K} \\
& \cdot \sin \left[\left(K+\frac{1}{2}\right) \alpha(g)\right]\left[1+0\left(\frac{1}{K}\right)\right]
\end{aligned}
$$

$$
A(g)=\left\{\left(\frac{\pi}{2}\right)^{2}+\left[\ln \left(\frac{\sqrt{h(g)-1}}{\sqrt{2 h(g)}+\sqrt{3 h(g)-1}}\right)-\frac{\sqrt{3 h(g)-1}}{3 \sqrt{2} g}\right]^{2}\right\}^{1 / 2}
$$




$$
\alpha(g)=\operatorname{arctg} \frac{\pi}{\frac{\sqrt{2(3 h-1)}}{3 g}+2 \ln \left(\frac{\sqrt{2 h}+\sqrt{3 h-1}}{\sqrt{h-1}}\right)}
$$

and the function $h(g)$ is defined by Eq. (2.7). Formulae (1.5)-(1.7) exactly coincide with the final results obtained by Hikami and Brézin [4] by a different procedure explained above.

In Sect. 4 we obtain the large orders in $1 / N$ for a non-symmetric model with lagrangian

$$
L(x)=\frac{1}{2}\left(\frac{d \vec{\phi}}{d x}\right)^{2}+\frac{\mu^{2}}{2} \sum_{a} C_{a}^{2} \phi_{a}^{2}+\frac{g}{N}\left(\vec{\phi}^{2}\right)^{2}
$$

where we assume that $L$ of the $C_{a}^{2}$ coefficients differ between them $(1 \leqq L \leqq N)$. For this non-symmetric model we find instantons that rule the large orders in $\frac{1}{N}$ by a similar procedure using SV. The stationary point equations in SV present $2^{L}-1$ solutions instead of one. They all correspond to reflectionless potentials with one, two, ... up to $L$ bound states. By inverse scattering techniques all instantons are expressed in closed form in terms of elementary functions [5].

Our final result reads

$$
I_{K}(g)=\sum_{s=1}^{2^{L}-1} \frac{\Gamma\left(K+\frac{M_{s}}{2}\right)}{\left[\rho_{s}(g)\right]^{K+M_{s} / 2}} C_{s}(g)\left[1+0\left(\frac{1}{K}\right)\right] .
$$

Here the index $s$ labels the $\left(2^{L}-1\right)$ different instantons and $M_{s}$ stands for the number of bound states in the $s^{\text {th }}$ instanton. The function $\rho_{s}(g)$ - the effective instanton action is given in Sect. 4 (Eqs. (4.17)-(4.18)).

The large $K$ limit in Eq. (1.9) will be dominated by the term of smallest $\left|\rho_{s}(g)\right|$. For small $g$ this is done by the instanton with one bound state (in SV) and smallest eigenvalue. On the contrary for very large $g$ it is also a one-bound instanton but with the largest eigenvalue which has smallest $\left|\rho_{s}\right|$. This is true at least for $1 \ll g^{1 / 3}>4 \frac{(L-1)}{N} \ln g$. In Sect. 4 we also discuss the case of a model with symmetry $0(P) \otimes 0(N-P)$.

In conclusion we have reduced the problem of large orders in $1 / N$ for the non-symmetric case to find the roots of a polynomial of degree $L$ (Eq. (4.9)). These roots are the possible bound states defining the instantons in SV. Then the effective instanton actions $\rho_{s}$ are given by (4.17)-(4.18). The action of a multi-bound states instanton simply adds because of complete separability.

\section{1/N Perturbation Theory and Inverse Scattering}

In this section we shall deal with the $N$-component anharmonic oscillator with $O(N)$ symmetry. Its generating function for imaginary time $(x)$ reads

$$
Z(N, g)=\int D \vec{\phi} \exp (-S[\vec{\phi}(\cdot)]) / Z_{0}
$$




$$
S[\vec{\phi}] \equiv \int_{-\infty}^{+\infty} d x\left[\frac{1}{2}\left(\frac{d \vec{\phi}}{d x}\right)^{2}+\frac{\mu^{2}}{2} \vec{\phi}^{2}+\frac{g \mu^{3}}{N}\left(\vec{\phi}^{2}\right)^{2}\right]
$$

where $\vec{\phi}(x)=\left(\phi_{1}, \ldots, \phi_{N}(x)\right), g$ is a dimensionless coupling constant and $\mu$ fixes the mass scale. $Z_{0}$ is a normalization constant fixed by $Z(N, 0) \equiv e^{-(N / 2) 2 T}$.

The expansion of $Z(N, g)$ in powers of $1 / N$ can be easily generated [1] on the basis of the identity

$$
\exp -\left\{\mu^{3} \frac{g}{N} \int d x\left(\vec{\phi}^{2}\right)^{2}\right\}=\iint D \alpha(.) \exp \left\{-\int d x \alpha(x)^{2}-2 i \mu^{3 / 2} \sqrt{\frac{g}{N}} \int \alpha(x) \vec{\phi}^{2} d x\right\}
$$

Upon replacing Eq. (2.3) into (2.1) the $\vec{\phi}$ integration turns out to be gaussian and it can be evaluated with the result

$$
\begin{aligned}
Z(N, g)= & \frac{1}{Z_{0}} \iint D \alpha(.) \exp \left\{-\frac{N}{2} \operatorname{Tr} \log \left[-\frac{d^{2}}{d x^{2}}+\mu^{2}+4 i \mu^{3 / 2} \sqrt{\frac{g}{N}} \alpha(.)\right]\right. \\
& \left.-\int d x \alpha(x)^{2}\right\} .
\end{aligned}
$$

This functional integral has a stationary point at $\alpha(x)=\alpha_{0}$, where

$$
4 i \mu^{3 / 2} \sqrt{\frac{g}{N}} \alpha_{0}+\mu^{2}=m^{2}
$$

and $m^{2}$ is the positive root of the equation

$$
\left(m^{2}-\mu^{2}\right) m-2 g \mu^{3}=0 .
$$

That is

$$
\begin{aligned}
& m^{2}=\mu^{2} h(g) \\
& h(g) \equiv \frac{2}{3}+\left[g+\sqrt{g^{2}-\frac{1}{27}}\right]^{2 / 3}+\left[g-\sqrt{g^{2}-\frac{1}{27}}\right]^{2 / 3}
\end{aligned}
$$

This gives in limiting cases

$$
\begin{aligned}
& h(g) \underset{g \downarrow 0}{=} 1+2 g-2 g^{2}+0\left(g^{3}\right) \\
& h(g) \underset{g \rightarrow+\infty}{=}(2 g)^{2 / 3}+\frac{2}{3}+0\left(g^{-2 / 3}\right) .
\end{aligned}
$$

The $1 / N$ expansion for $Z(N, g)$ follows by expanding the integrand around this constant stationary point

$$
Z(N, g)=\left(\frac{m}{\mu}\right)^{N / 4} \exp \left\{-\frac{N}{2}\left(m-\frac{\mu g}{2 h}\right)(2 T)\right\} \int D \alpha(\cdot) \exp \left(-S_{\text {eff }}[\alpha(\cdot)]\right)
$$


Here

$$
\begin{aligned}
S_{\text {eff }}[\alpha(\cdot) ; N]= & \frac{N}{2}\left\{\operatorname{Tr} \log \left(\frac{-\frac{d^{2}}{d x^{2}}+m^{2}+v(\cdot)}{-\frac{d^{2}}{d x^{2}}+m^{2}}\right)-\frac{1}{8 g \mu^{3}} \int d x v(x)^{2}\right. \\
& -\frac{m^{2}-\mu^{2}}{4 g \mu^{3}} \int d x v(x)
\end{aligned}
$$

$T$ is a large cutoff in the $x$ coordinate and we have set

$$
\alpha(x)=\alpha_{0}+\frac{1}{4 i \mu^{3 / 2}} \sqrt{\frac{N}{g}} v(x) .
$$

The ground state energy reads

$$
\begin{aligned}
E_{G} & =-\lim _{T \rightarrow \infty} \frac{1}{2 T} \log Z(N, g) \\
& =\frac{N}{2}\left(m-\frac{\mu g}{2 h}\right)-[\sqrt{3 h(g)-1}-\sqrt{2 h(g)}] \frac{\mu}{\sqrt{2}}+\sum_{K=1}^{\infty} \frac{A_{K}(g)}{N^{K}}
\end{aligned}
$$

The coefficients $A_{K}(g)$ can be computed as sum of Feynman diagrams with propagators

$$
\Delta(P)=\frac{4 \mu^{3} g}{m\left(P^{2}+4 m^{2}\right)}
$$

as lines and vertices coming from functionals derivatives of $S_{\text {eff }}$ at $\alpha(x)=\alpha_{0}$ of order larger or equal to three. We recall that $\left.\delta^{L} S_{\text {eff }}\right|_{\alpha_{0}}=0\left(N^{1-L / 2}\right)$.

We are interested on the behavior of the coefficients $A_{K}(g)$ for large $K$ at $g$ fixed. For $K \gg 1, A_{K}(g)$ has the same behavior as the integral

$$
\begin{aligned}
& I_{K}(g) \equiv-\lim _{T \rightarrow \infty} \frac{1}{2 T} \oint \frac{d N}{2 \pi i} N^{K-1} \frac{\iint D \alpha(\cdot) e^{-S_{\text {eff }\{\alpha(\cdot) N\}}}}{\iint D \alpha(\cdot) e^{-\left(\alpha\left|M_{0}\right| \alpha\right)}} \\
& A_{K}(g)=\underset{K \rightarrow \infty}{=} I_{K}(g)\left[1+0\left(\frac{1}{K}\right)\right]
\end{aligned}
$$

Here

$$
\left.M_{0}(x, y) \equiv \frac{1}{2} \frac{\delta^{2} S_{\mathrm{eff}}}{\delta \alpha(x) \delta \alpha(y)}\right|_{\alpha \cdot \cdot)=\alpha_{c}}=\delta(x-y)+\frac{g \mu^{3}}{m^{2}} e^{-2 m|x-y|}
$$

Now, we proceed "à la Lipatov" $[2,3]$ and seek for stationary points in (2.15)

$$
0=\frac{\delta S_{\mathrm{ef}}}{\delta v(x)}=\frac{N}{2}\left\{\left\langle x\left|\frac{1}{-\frac{d^{2}}{d x^{2}}+m^{2}+v(\cdot)}\right| x\right\rangle-\frac{v(x)+m^{2}-\mu^{2}}{4 g \mu^{3}}\right\}
$$

with boundary conditions $v( \pm \infty)=0$. 
It is not clear for us how to get $v(x)$ from Eq. (2.18) as it stands. However, the equation for the instanton is much simpler in another variables. They are the inverse scattering data associated with $v(x)$ if we regard it as a quantum potential [5].

That is, we consider the auxiliar Schroedinger equation

$$
\left[-\frac{d^{2}}{d x^{2}}+v(x)\right] \psi_{(x, k)}=k^{2} \psi_{(x, k)} .
$$

We define as usual the inverse scattering variables $\mathrm{SV}$ as the set

$$
S V=\left\{r(k),-\infty<k<+\infty ; \kappa_{l}, C_{l}, l=1, \ldots, N_{B}\right\} .
$$

Here $r(k)$ is the reflection coefficient, $-\kappa_{l}^{2}$ the eigenvalue of the $l^{\text {th }}$ bound state and $C_{l}$ the normalization coefficient of the corresponding wave function. $N_{B}$ stands for the total number of bound states $\left(N_{B} \geqq 0\right)[5]$.

As it is known there is a one-to-one correspondence between potentials $v(x)$ and sets $S V$ under some regularity conditions [5]. Thus we can get stationary points by writing the effective action in terms of the $S V$ and varying them. The crucial advantage of the $S V$ will be here the fact that the effective action completely separates in the new variables.

The functional determinant in $S_{\text {eff }}$ [Eq. (2.11)] coincides with the Jost function associated with the Schroedinger equation (2.19). This Jost function $F\left[k^{2} ; v(\cdot)\right]$, as well as the integrals involving $v(x)$ in Eq. (2.11) can be expressed in terms of $S V$ as

$$
\begin{aligned}
& F\left[k^{2} ; v(\cdot)\right]=\prod_{j=1}^{N_{B}}\left(\frac{k-i \kappa_{j}}{k+i \kappa_{j}}\right) \exp \left(\frac{1}{2 \pi i} \int_{-\infty}^{+\infty} \frac{d k^{\prime}}{k-k^{\prime}+i 0} \ln \left[1-\left|r\left(k^{\prime}\right)\right|^{2}\right]\right) \\
& \int_{-\infty}^{+\infty} d x v(x)=-\frac{1}{\pi} \int_{-\infty}^{+\infty} d k \ln \left[1-|r(k)|^{2}\right]-4 \sum_{j=1}^{N_{B}} \kappa_{j} \\
& \int_{-\infty}^{+\infty} d x v(x)^{2}=-\frac{4}{\pi} \int_{-\infty}^{+\infty} k^{2} d k \ln \left[1-|r(k)|^{2}\right]+\frac{16}{3} \sum_{j=1}^{N_{B}} \kappa_{j}^{3}
\end{aligned}
$$

Eqs. (2.22-3) are the two first trace identities associated to the Korteweg-de Vries equation [7]. Finally, from Eqs. (2.11)-(2.21-23) we get for the effective action in the $S V$

$$
\begin{aligned}
S_{\text {eff }}[\alpha(\cdot) ; N]= & \frac{N}{2}\left\{-\sum_{j=1}^{N_{B}}\left[2 \operatorname{Arg} \operatorname{Th}\left(\frac{\kappa_{j}}{m}\right)+\frac{\kappa_{j}}{g \mu^{3}}\left(\frac{2}{3} \kappa_{j}^{2}+\mu^{2}-m^{2}\right)\right]\right. \\
& \left.+\int_{-\infty}^{+\infty} \frac{d k}{2 \pi}\left[\frac{i}{k-i m}+\frac{2 k^{2}+m^{2}-\mu^{2}}{2 g \mu^{3}}\right] \ln \left[1-|r(k)|^{2}\right]\right\}
\end{aligned}
$$

We find $S_{\text {eff }}$ independent of the normalization coefficients $C_{l}$. This comes from the translational invariance of $S_{\text {eff }}$ in the variables $\alpha(x)$. The extrema of the effective action now follows from

$$
\frac{\delta S}{\delta r(k)}=0 \quad-\infty<k<+\infty
$$




$$
\frac{\delta S}{\partial \kappa_{j}}=0 \quad 1 \leqq j \leqq N_{B}
$$

The first one has as unique solution

$$
r(k)=0 \quad \text { for all } k
$$

The second equation gives

$$
\kappa_{j}^{2}\left[\kappa_{j}^{2}-\frac{3 m^{2}-\mu^{2}}{2}\right]=0
$$

In consequence, we have the non trivial solution

$$
\begin{array}{ll}
\kappa=\sqrt{\frac{3 m^{2}-\mu^{2}}{2}} & N_{B}=1 \\
r(k)=0, & -\infty<k<+\infty
\end{array}
$$

In the former variables this is a reflectionless potential $v_{c}(x)$ with only one bound state at $k^{2}=-\kappa^{2}$. It is easy to reconstruct $v_{c}(x)$ from the Gelfand-LevitanMarchenko [5] equation, with the result

$$
v_{c}(x)=-\frac{2 \kappa^{2}}{\cosh ^{2} \kappa x}
$$

The trivial solution of Eq. (2.28) corresponds to the constant extremum $\alpha(x)=\alpha_{0}$.

The effective action at the extremum (2.29) takes the value

$$
\begin{aligned}
& S_{ \pm}=N \rho_{ \pm}(g) \\
& \rho_{ \pm}(g)=\frac{1}{2} \log [h(g)-1]-\log [\sqrt{2 h(g)}+\sqrt{3 h(g)-1}] \\
& -\frac{1}{3 \sqrt{2} g} \sqrt{3 h(g)-1} \pm \frac{i \pi}{2}
\end{aligned}
$$

The sign + or - depends on the choice $\frac{\kappa}{m} \pm i 0$ in Eq. (2.24). Both signs lead to extrema of the action. So, we must sum both contributions obtaining in this way a real result for $A_{K}(g)$ as it should be.

\section{Large Orders of the $1 / N$ Perturbative Series}

We compute in this section the small fluctuations around the instanton $v_{c}(x)$ found in the preceding reaction. In this way we obtain $A_{K}(g)$ for large $K$ up to corrections of order $K^{-1}$.

Our starting point is Eq. (2.16) where we expand $S_{\text {eff }}$ up to second order around $\alpha_{c}(x)$.

This can be written as

$$
\begin{aligned}
& A_{K}(g)=-\lim _{T \rightarrow \infty}\left\{\frac{1}{2 T} \int_{\mathbb{C}} \frac{d N}{2 \pi i} N^{K-1} \exp \left\{-N \rho_{+}(g)\right\}\right. \\
& \left.\cdot \frac{\iint D \alpha(\cdot) \exp \left(-\left\langle\alpha\left|M_{c}\right| \alpha\right\rangle\right)}{\iint D \alpha(\cdot) \exp \left(-\left\langle\alpha\left|M_{0}\right| \alpha\right\rangle\right)}+C . C .\right\}\left\{1+O\left(K^{-1}\right)\right\}
\end{aligned}
$$


Here, $\mathbb{C}$ is a contour in the $N$-plane that begin at $N=-\infty-i \varepsilon$, encircles the origin counter-clockwise and ends at $N=-\infty+i \varepsilon . M_{C}$ stands for the small fluctuations matrix around $\alpha_{c}(x)$

$$
\left.M_{c}\left(x, x^{\prime}\right) \equiv \frac{1}{2} \frac{\delta^{2} S_{\text {eff }}}{\delta \alpha(x) \delta \alpha\left(x^{\prime}\right)}\right|_{\alpha=\alpha_{c}(x)}=\delta\left(x-x^{\prime}\right)+4 g \mu^{3} G_{c}\left(x, x^{\prime}\right)^{2}
$$

Here $G_{c}\left(x, x^{\prime}\right)$ is the Green's function of the Schroedinger equation with $v_{c}(x)$ as potential

$$
\begin{aligned}
G_{c}\left(x, x^{\prime}\right) & \equiv\left\langle x\left|\frac{1}{-\frac{d^{2}}{d x^{2}}+m^{2}+v_{c}(\cdot)}\right| x^{\prime}\right\rangle= \\
& =-\frac{e^{-m\left|x-x^{\prime}\right|}}{m\left(m^{2}-\mu^{2}\right)}\left[m+\kappa t h \kappa x_{>}\right]\left[m-\kappa t h \kappa x_{<}\right] .
\end{aligned}
$$

In the derivation we used the relation

$$
\frac{\delta}{\delta v(y)} G\left(x, x^{\prime}\right)=-G(x, y) G\left(y, x^{\prime}\right)
$$

One can see immediately from eq. (3.3) that $v_{c}(x)$ verifies the instanton equation in $v$ variables [Eq. (2.18)].

The small fluctuations matrix $M_{c}(x, y)$ has a zero mode associated to the translational invariance of the effective action. That is

$$
\int_{-\infty}^{-\infty} M_{c}(x, y) \frac{d v_{c}}{d y} d y=0
$$

The presence of this zero mode tell us that we must extract a collective coordinate [8] associated to translations in the functional integral (3.1). After this change of integration variables the functional integral reads for large $T$

$$
\frac{\iint D \alpha(\cdot) e^{-\left(\alpha\left|M_{c}\right| \alpha\right)}}{\iint D \alpha(\cdot) e^{-\left(\alpha\left|M_{0}\right| \alpha\right)}}=\sqrt{\frac{S_{c}}{\pi}} 2 T \sqrt{\frac{\lambda_{S}(T)}{\operatorname{det}\left(\frac{M_{C}}{M_{0}}\right)}}
$$

where

$$
S_{c}=\int_{-\infty}^{+\infty} d x \alpha_{c}^{\prime}(x)^{2}=-\frac{N \mu^{2}}{15 \sqrt{2 g}}\left(\frac{3 m^{2}}{\mu^{2}}-1\right)^{5 / 2}
$$

and $\lambda_{s}(T)$ is a small eigenvalue of the operator $M_{c}$ that vanishes for $T \rightarrow \infty$. The computation of the infinite determinant in eq. (3.5) and of $\lambda_{s}(T)$ can be greatly simplified by noting that $G_{c}\left(x, x^{\prime}\right)^{2}$ and $G_{0}\left(x, x^{\prime}\right)^{2}$ have the structure of one dimensional Green functions. That is, they factorize into a function of $x_{<}$times another function of $x_{>}$. Hence, one can construct second order differential operators $\hat{\Sigma}_{c}$ and $\hat{\Sigma}_{0}$ for whom, $G_{c}^{2}\left(x, x^{\prime}\right)$ and $G_{0}^{2}\left(x, x^{\prime}\right)$ are their respective Green functions. 
The explicit expressions for them are

$$
\begin{aligned}
\left\langle x\left|\hat{\Sigma}_{c}\right| x^{\prime}\right\rangle= & +\delta\left(x-x^{\prime}\right)\left\{-\frac{d}{d x^{\prime}}\left[\frac{1}{\kappa^{2} t h^{2} \kappa x^{\prime}-m^{2}} \frac{d}{d}\right]\right. \\
& \left.+2+\frac{2\left(m^{2}-\mu^{2}\right)\left(3 m^{2}+\mu^{2}\right)}{\left[\left(3 m^{2}-\mu^{2}\right) t h^{2} \kappa x^{\prime}-2 m^{2}\right]^{2}}\right\} \\
\left\langle x\left|\hat{\Sigma}_{0}\right| x^{\prime}\right\rangle= & \delta\left(x-x^{\prime}\right)\left[-\frac{d^{2}}{d x^{\prime 2}}+4 m^{2}\right] .
\end{aligned}
$$

It follows from (2.17) and (3.2) that

$$
\begin{aligned}
& \hat{\Sigma}_{c} \hat{M}_{c}=\hat{\Sigma}_{c}+4 \\
& \hat{\Sigma}_{0} \hat{M}_{0}=\hat{\Sigma}_{0}+4\left(\kappa^{2}-m^{2}\right) \equiv \hat{\Sigma}_{0}^{\prime} .
\end{aligned}
$$

In this way we get

$$
\operatorname{det}\left(\frac{M_{c}}{M_{0}}\right)=\frac{\operatorname{det}\left(\frac{\Sigma_{c}+4}{\Sigma_{0}^{\prime}}\right)}{\operatorname{det}\left(\frac{\Sigma_{c}}{\Sigma_{0}}\right)} \underset{T \rightarrow \infty}{=} 2\left(\frac{m+\kappa}{m-\kappa}\right)^{2} e^{-4 \kappa T}
$$

where, in the last step, we have used Van Vleck's formula [9].

The operator $\hat{\Sigma}_{c}$ is also useful to compute the small eigenvalue $\lambda_{s}(T)$. If we call $\psi_{s}$ to its corresponding wave functions

$$
\hat{M}_{c} \psi_{s}=\lambda_{s} \psi_{s}
$$

and we apply $\hat{\Sigma}_{c}$ on both sides we find

$$
\left(\hat{\Sigma}_{c}+4\right) \psi_{s}=-\frac{4 \lambda_{s}}{1-\lambda_{s}} \psi_{s} \underset{T \rightarrow \infty}{\simeq}-4 \lambda_{s} \psi_{s}
$$

Then by wronskian arguments applied to the differential operator $\left(\hat{\Sigma}_{c}+4\right)$ one easily finds for large $T$

$$
\lambda_{s}(T)=120 \frac{3 h(g)-1}{h(g)-1} e^{-4 k T}
$$

This completes our calculation of the functional integrals over $\alpha(\cdot)$ in Eq. (3.1) or (3.5). From eqs. (3.1), (3.5), (3.6), (3.10) and (3.13) we obtain finally, upon integrating over $N$

$$
\begin{aligned}
A_{K}(g)= & \mu\left\{\frac{\Gamma\left(K+\frac{1}{2}\right) 2^{3 / 4}}{\rho_{+}^{K+1 / 2}} \frac{[3 h(g)-1]^{7 / 4}}{\pi^{3 / 2} \sqrt{g[h(g)-1]}} \frac{\sqrt{3 h-1}-\sqrt{2 h}}{\sqrt{3 h-1}+\sqrt{2 h}}+C . C\right\} \\
& \cdot\left[1+0\left(\frac{1}{K}\right)\right]
\end{aligned}
$$

The two terms here can be combined as it is shown in Sect. 1 Eq. (1.5). 


\section{Large Orders in the $1 / N$ Expansion for a Non-Symmetric $O(N)$ Model}

In this section we find the large order behavior of the $1 / N$ expansion in the anharmonic oscillator when the mass term breaks the $0(N)$ symmetry. That is

$$
L(x)=\frac{1}{2}\left(\frac{d \vec{\phi}}{d x}\right)^{2}+\frac{\mu^{2}}{2} \sum_{a=1}^{N} C_{a}^{2} \phi_{a}^{2}+\frac{g \mu^{3}}{N}\left(\vec{\phi}^{2}\right)^{2}
$$

Here the $C_{a}^{2}\left(C_{1}^{2} \leqq C_{2}^{2} \leqq \ldots \leqq C_{N}^{2}\right)$ are positive dimensionless parameters of order one. We assume that $L$ of them are inequal and we denote by $\left\{d_{\alpha}^{2}, \alpha=1, \ldots, L\right\}$ the set of different $C_{a}^{2}$ parameters $\left(d_{1}^{2}<d_{2}^{2}<\ldots<d_{L}^{2}\right)$. $(1 \leqq L \leqq N)$.

Following the same procedure as in Sect. 3 we can write the generating function for this model as

$$
\begin{aligned}
& Z(N, g)=\frac{1}{Z_{0}} \iint D \alpha(\cdot) \exp \left\{-S_{\text {eff }}[\alpha(\cdot), N]\right\} \\
& S_{\text {eff }}[\alpha(\cdot), N]=\frac{1}{2} \sum_{a=1}^{N} \operatorname{Tr} \log \left[-\frac{d^{2}}{d x^{2}}+\mu^{2} C_{a}^{2}+4 i \mu^{3 / 2} \sqrt{\frac{g}{N}} \alpha(\cdot)\right]+\int d x \alpha(x)^{2} .
\end{aligned}
$$

This functional integral has a constant stationary point at

$$
\alpha(x)=\alpha_{B} \equiv \frac{1}{4 i} \sqrt{\frac{\mu N}{g}} z(g)
$$

where $z(g)$ is the positive solution of the algebraic equation

$$
z-\frac{2 g}{N} \sum_{\alpha=1}^{N} \frac{1}{\sqrt{z+C_{a}^{2}}}=0
$$

which reduces to Eqs. (2.5)-(2.6) in the $0(N)$ symmetric case. It is easy to check that Eq. (4.5) has one and only one positive solution for $0<g<\infty$, with the following limiting behavior

$$
\begin{aligned}
& z(g) \underset{g \downarrow 0}{=} 2 g\left(\frac{1}{N} \sum_{a=1}^{N} \frac{1}{C_{a}}\right)\left[1-\frac{g}{N} \sum_{b=1}^{N} \frac{1}{C_{b}^{3}}+O\left(g^{2}\right)\right] \\
& z(g) \underset{g \backslash \infty}{=}(2 g)^{2 / 3}-\frac{1}{2 N} \sum_{a=1}^{N} C_{a}^{2}+O\left(g^{-2 / 3}\right) .
\end{aligned}
$$

The $1 / N$ series for the broken $-0(N)$ model can be obtained by expanding the effective action in the path integral (4.2) around $\alpha_{B}$. Following similar lines to those of section 2 the ground state energy turns out to be here

$$
E_{G}=\frac{\mu}{2} \sum_{a=1}^{N} \sqrt{z+C_{a}^{2}}-\frac{\mu N z^{2}}{16 g}+\mu \sum_{\alpha=1}^{L}\left(t_{\alpha}-\sqrt{1+d_{\alpha}^{2}}\right)+\sum_{K=1}^{\infty} \frac{A_{K}(g, N)}{N^{k}} .
$$

Here the $t_{\alpha}$ are the roots of the algebraic equation

$$
1+\frac{g}{N} \sum_{a=1}^{N} \frac{1}{\sqrt{z+C_{a}^{2}}\left[z+C_{a}^{2}-t^{2}\right]}=0 .
$$


This equation has $L$ different positive roots $\left(t_{1}, \ldots, t_{L}\right)$. It must be noted that the coefficients $A_{K}$ now depend explicitly on $N$ but this is always through expressions like

$$
\frac{1}{N} \sum_{a=1}^{N} Z_{a}
$$

where the $Z_{a}$ are of order $N^{0}$. Thus, the $A_{K}(g, N)$ are also of order $N^{0}$. For example the propagator reads here

$$
\Delta(P)=\frac{4 \mu^{2} g}{N} \sum_{a=1}^{N} \frac{1}{\sqrt{z+C_{a}^{2}}\left[P^{2}+4 \mu^{2}\left(z+C_{a}^{2}\right)\right]}
$$

Let us now study the large $K$ behavior of the $A_{K}(g, N)$. We do that as in section 2 from the generalization of eq. (2.15). We take for a moment another variable, say $N^{\prime}$, instead of $N$ inside $A_{K}$ and we consider $N^{\prime}$ fixed and independent of $N$.

As before we find instantons as reflectionless potentials but now with a richer set of possible bound states. The equation for the instantons

$$
\frac{\partial S_{\text {eff }}}{\partial \kappa_{j}}=0, \quad 0 \leqq j \leqq N_{B}
$$

gives now eq. (4.9) with $\kappa_{j}^{2}=\mu^{2} t_{j}^{2}$. We find then $L$ different solutions for the possible bound state energies and hence $2^{L}-1$ different instantons by doing all possible combinations of energy levels:

$L \quad$ potentials with one bound state

$\frac{L(L-1)}{2}$ potentials with two bound states

$\left(\begin{array}{c}L \\ M\end{array}\right) \quad$ potentials with $M$ bound states
$\ldots \cdots$
$1 \quad$ potential with $L$ bound states

All these instantons can be expressed in a closed form in terms of elementary functions [5]. They are $M$-soliton solutions of the K.d.V. equation at initial time.

Limiting expressions for the bound state energies at small and large $g$ respectively read

$$
\begin{aligned}
& t_{\alpha}^{2}=C_{\alpha}^{2}+g\left(\frac{2}{N d_{\alpha}}+\frac{1}{N} \sum_{b} \frac{1}{C_{b}}\right)+O\left(g^{2}\right), 1 \leqq \alpha \leqq L \\
& t_{\alpha}^{2}=(2 g)^{2 / 3}+D_{\alpha}^{2}+O\left(g^{-2 / 3}\right), 1 \leqq \alpha \leqq L-1 \\
& t_{L}^{2}=\frac{3}{2}(2 g)^{2 / 3}-\frac{1}{2 N} \sum_{a=1}^{N} C_{a}^{2}-\frac{1}{4 N} \sum_{b=1}^{N} \frac{1}{C_{b}^{2}-\frac{1}{2 N} \sum_{a} C_{a}^{2}}+O\left(g^{-2 / 3}\right)
\end{aligned}
$$

where the $D_{\alpha}$ are the positive solutions of the algebraic equation

$$
\sum_{a=1}^{N} \frac{1}{C_{a}^{2}-D^{2}-\frac{1}{2 N} \sum^{N} C_{b}^{2}}=0
$$


and we assume that the $C_{a}^{2}$ are such that the denominators in eqs. (4.13) and (4.14) do not vanish.

Moreover the following inequalities hold for any positive $g$

$$
d_{1}^{2}+z(g)<t_{1}^{2}(g)<d_{2}^{2}+z(g)<\ldots<d_{L}^{2}+z(g)<t_{L}^{2}(g) \text {. }
$$

The contribution of each stationary point to $A_{K}\left(g, N^{\prime}\right)$ follows by the same methods as in Sect. 3. In the present case a $M$-bound state potential presents $M$ zero modes and the jacobian of the collective coordinates is then a $M \times M$ determinant proportional to $N^{M / 2}$. Because of this a $M$-bound state instanton $\alpha_{s}(x)$ contributes to $A_{K}\left(g, N^{\prime}\right)$ by

$$
\frac{\Gamma\left(K+\frac{M}{2}\right)}{\rho_{s}(g)^{K+M / 2}} C_{s}\left(g, N^{\prime}\right)\left[1+0\left(\frac{1}{K}\right)\right] K \gg 1
$$

where $C_{s}\left(g, N^{\prime}\right)$ (independent of $K$ ) is determined by the small fluctuations determinant around $\alpha_{s}(x)$ and $\rho_{s}(g)$ is the sum of $M$ terms like

$$
\begin{aligned}
F \alpha(g)= & \pm \frac{i \pi n(\alpha)}{2 N}-\frac{1}{N}\left[\sum_{a=1}^{n(\alpha)} \operatorname{Arg} \operatorname{Th}\left(\frac{\sqrt{C_{a}^{2}+z(g)}}{t_{\alpha}(g)}\right)+\right. \\
& \left.+\sum_{a=n(\alpha)+1}^{N} \operatorname{Arg} \operatorname{Th}\left(\frac{t_{\alpha}(g)}{\sqrt{C_{a}^{2}+z(g)}}\right)\right]-\frac{t_{\alpha}(g)}{6 g}\left[2 t_{\alpha}(g)^{2}-3 z(g)\right] .
\end{aligned}
$$

Here $n(\alpha)$ is the total number of $C_{a}^{2}$ with $C_{a}^{2} \leqq d_{\alpha}^{2}$. That is

$$
\rho_{s}(g)=\sum_{\alpha} F_{\alpha}(g)
$$

where $\alpha$ runs over the $M$ bound states associated to $\alpha_{s}(x)$.

The large $K$ behavior of $A_{K}\left(g, N^{\prime}\right)$ will be given by (4.16) for the $\alpha_{s}(x)$ with smallest $\left|\rho_{s}(g)\right|$.

Let us analyse the limits $g \rightarrow 0$ and $g \rightarrow \infty$. For small $g$ we find

$$
F_{\alpha}(g)=\frac{C_{\alpha}^{3}}{3 g}-\frac{1}{2} \ln g+0(1)
$$

Then, the one-bound state stationary point with the smallest eigenvalue, $\kappa_{1}^{2}=\mu^{2} t_{1}^{2}$, dominates the large $K$ behavior of $A_{K}\left(g, N^{\prime}\right)$. We have for strong coupling

$$
\begin{aligned}
& F_{\alpha}(g) \underset{g \uparrow \infty}{=} \frac{1}{6} g^{1 / 3}-\frac{L-1}{3 N} \ln g+0(1), \quad 1 \leqq \alpha \leqq L-1 \\
& F_{L}(g) \underset{g \uparrow \infty}{=}-\frac{L-1}{3 N} \ln g+0(1) .
\end{aligned}
$$

In the extreme large $g$ limit we find that the dominant contribution to $A_{K}\left(g, N^{\prime}\right)$ comes from the instanton associated to the bound state of largest eigenvalue $\left(t_{L}^{2}\right)$. This is true at least for

$$
1 \ll g^{1 / 3}>4 \frac{(L-1)}{N} \ln g .
$$


For smaller values of $g$ the situation is not so simple because the smallest $\rho_{s}$ could correspond in principle to an instanton with several bound states $\left(t_{L}^{2}\right.$ among them).

It should be noted that the effective actions never vanish. We have the lower bound

$$
\left|\rho_{s}(g)\right| \geqq\left|\operatorname{Im} \rho_{s}(g)\right| \geqq \frac{\pi}{2}
$$

As a last example we consider the case of two different masses with arbitrary $N$. That is

$$
C_{1}^{2}=C_{2}^{2}=\ldots=C_{P}^{2}=d_{1}^{2}=1 \text { and } C_{P+1}^{2}=\ldots=C_{N}^{2}=d_{2}^{2}=b^{2}>1
$$

where $P$ is some integer $1<P<N$. This a model with $0(P) \otimes 0(N-P)$ symmetry. We can easily discuss two limiting cases

$$
0<b^{2}-1 \ll 1 \text { and } b^{2} \gg 1
$$

for arbitrary values of $g$.

If $b^{2}-1 \ll 1$ the instanton effective actions read

$$
\begin{aligned}
& F_{1}(g)= \pm \frac{i \pi P}{2 N}-\log \left(\frac{4 h(g)}{b^{2}-1}\right)+\ln \left[\left(\frac{P}{N}\right)^{P / N}\left(1-\frac{P}{N}\right)^{P / N}\right]+0\left(b^{2}-1\right) \\
& F_{2}(g)=\rho_{ \pm}(g)+0\left(b^{2}-1\right) .
\end{aligned}
$$

In the other limiting case $b^{2} \gg 1$ and fixed $g$ we find

$$
\begin{aligned}
& F_{1}(g)=\frac{P}{N} \rho_{ \pm}\left(\frac{P}{N} g\right)+0(1 / b) \\
& F_{2}(g)= \pm \frac{i \pi}{2}-\frac{b^{2}}{3 g}+\frac{h\left(\frac{g P}{N}\right)-1}{2 g} b-\left(1-\frac{P}{N}\right) \log b+0(1)
\end{aligned}
$$

For all $b$ we have for the two-bound state instanton

$$
F_{3}(g)=F_{1}(g)+F_{2}(g) \text {. }
$$

The $1 / N$ expansion for large orders behave as in a model with symmetry $O(N)$ if $b^{2} \downarrow 1$ and symmetry $0(P)$ if $b \uparrow \infty$. Moreover we see explicitly how the nondominant contributions decreases when we approach the symmetry limits.

Acknowledgements. I wish to thank Edouard Brézin and J. Zinn-Justin for many fruitful discussions and encouragement. I thank the members of the Service of Physique Théorique at Saclay for the kind hospitality extended to me.

\section{References}

1. Ma, S. K. : Modern theory of critical phenomena. (ed. W. A. Benjamin), Massachusetts (1976); Brézin, E., Le Guillou, J. C., Zinn-Justin, J. : Field theoretical approach to critical phenomena. In: Phase transitions and critical phenomena. (Eds. C. Domb, M. S. Green). Academic Press: London 1976 
2. Lipatov, L. N. : ZhETF Pis. Red. 25, 116 (1977)

(JETP Letters 25, 105 (1977)); ZhETF 72, 411 (1977) [JETP 45, 216 (1977)]

3. Brézin, E., Le Guillou, J. C., Zinn-Justin, J. : Phys. Rev. D15, 1544 and 1558 (1977)

4. Hikami, S., Brézin, E. : Saclay preprint DPH-T/78/76

5. Scott, A. C., Chu, F. Y. F., McLaughlin, D. W. : Proc. of the IEEE 61, 1443 (1973)

Chadan, K., Sabatier, D. C. : Inverse problems in quantum scattering theory. Berlin, Heidelberg, New York: Springer (1977)

Inverse scattering techniques was used in QFT by several authors. See for example Dashen, R. F., Hasslacher, B., Neveu, A. : Phys. Rev. D12, 2443 (1975)

Korepin, V. E., Kulish, P. P., Faddeev, L. D. : ZhETF Pis. Red. 21, 302 (1975) [JETP Letter 21, $138(1975)]$

Gervais, J. L., Neveu, A., Virasoro, M. A. : Nucl. Phys. B138, 45 (1978)

6. Choodnovsky, D. V., Choodnovsky, G. V.: Seminaire sur les équations non-linéaires. Ecole Polytechnique (1977-1978)

7. Faddeev, L. D., Zajarov, V. E. : Funk. Anal. Eg. Pri. 5, 18 (1971) [Funct. Anal. Appl. 5, 280 (1971)]

8. Gervais, J. L., Jevicki, A., Sakita, B. : In: Proceedings of the Paris Conference, (eds. J. L. Gervais and A. Neveu) Phys. Rev. 23C, 240 (1976)

9. Gelfand, I. M., Yaglom, A. M. : J. Math. Phys. 1, 48 (1960)

Dashen, R. F., Hasslacher, B., Neveu, A. : Phys. Rev. D10, 4114 (1974)

Communicated by E. Brézin

Received February 6, 1979 